\section{P1-161 THE BURDEN OF CANCER: AN APPROACH TO EVALUATE IN-HOSPITAL PROFILE OF COST IN ITALY}

doi:10.1136/jech.2011.142976d.54

${ }^{1} \mathrm{~A}$ Gigli, ${ }^{2} \mathrm{E}$ Crocetti, ${ }^{3} \mathrm{~S}$ Francisci, ${ }^{2} \mathrm{~F}$ Giusti, ${ }^{4} \mathrm{~S}$ Guzzinati, ${ }^{5} \mathrm{M}$ Mezzetti, ${ }^{2} \mathrm{G}$ Miccinesi, ${ }^{2} \mathrm{E}$ Paci, ${ }^{4} \mathrm{P}$ Zambon. ${ }^{1}$ Istituto di Ricerche sulla Popolazione e le Politiche Sociali, CNR, Roma, Italy; ${ }^{2}$ Registro Tumori della Regione Toscana, Firenze, Italy; ${ }^{3}$ Istituto Superiore di Sanità, Roma, Italy; ${ }^{4}$ Registro Tumori Veneto, Padova, Italy; ${ }^{5}$ Facoltà di Economia, Università di Tor Vergata, Roma, Italy

Introduction Due to changes in cancer-related risk factors, improvements in diagnostic procedures and treatments, and the ageing of the population in most developed countries, cancer accounts for an increasing proportion of healthcare expenditures. Measuring the burden of disease is of great interest to public health researchers and policy makers. The objective of this study is: (1) to reconstruct the cancer pathway, which is subdivided into three phases according to the disease and cost-related dynamics: initial (1 year after diagnosis), continuing (time between initial and final) and final (1 year before death); (2) to estimate the related cost subdivided into phases of care. Methods The profiles of cost directly attributable to cancer are reconstructed, using hospital discharge cards and DRG codes, in a cohort of colorectal cancer cases diagnosed in two areas covered by cancer registries in Italy in years 2000-2001 and followed-up to end of 2006; cancer survivors at end of 2005 in the two areas are decomposed into the three phases of care; and are multiplied by the corresponding cost profiles, to obtain an estimate of the in-hospital cost of care subdivided into phases of care.

Results Cost distribution curve follows a U-shape: higher initial and final cost and lower cost in the continuing phase. There is a trend by age and stage at diagnosis.

Conclusion Data confirm the connection between stage at diagnosis, profile of therapies and related cost. Results show that primary prevention and early detection of cancer are extremely important in a public health perspective.

\section{P1-162 PLANNED CAESAREAN SECTION ASSESSMENT IN AN ITALIAN REGION}

doi:10.1136/jech.2011.142976d.55

${ }^{1} \mathrm{~L}$ Minelli, ${ }^{1} \mathrm{M}$ Chiavarini, ${ }^{1} \mathrm{I}$ Bernardini, ${ }^{2} \mathrm{~A}$ Gili.. ${ }^{1}$ Department of Medical and Surgery Specialities and Public Health, University of Perugia, Italy, Perugia, Umbria, Italy; ${ }^{2}$ Department of Economics, Finance and Statistics, University of Perugia, Italy, Perugia, Umbria, Italy

Introduction This study aimed to evaluate the impact of planned caesarean section (PCS) and investigate variation in its use in 11 hospital maternity services in the Umbria region (Central Italy).

Methods A population-based study using routine maternity discharge data (CEDAP) from 2007 to 2008. The study included 15898 women (nulliparous, single cephalic, $\geq 37$ weeks, physiological pregnancy and without fetal development defects). The outcome of the study was the "planned caesarean" section rate and excluded women with "emergency caesarean". Using a multi-level model we evaluated non-observed heterogeneity; the second level variable was represented by different birthing centres and two context variables were included: the presence or absence of neonatal intensive care unit and the capacity of the service (1000 birth per year).

Results Of the 6811 births, 5934 (87.12\%) were born by natural labour and $877(12.88 \%)$ by PCS. The logistic model with random effects including some control variables at the individual level indicated a significant value in the residual variance of the second level ( $\rho$ 0.086). Introducing the two context variables resulted in a substantial reduction in unexplained variance.

Conclusions Variation in PCS rates in the 11 birthing centres is explained by the presence or absence of a neonatal intensive care unit and by birth centre capacity. These findings can be used by policy-makers to increase the efficiency and availability of appropriate technology in hospital maternity services.

\section{P1-163 INCREASED INCIDENCE AND DETECTION RATES OF CARCINOMA IN SITU OF THE BREAST}

doi:10.1136/jech.2011.142976d.56

${ }^{1} \mathrm{~J}$ A Glover, ${ }^{1} \mathrm{C}$ M Hughes, ${ }^{2} \mathrm{M} \mathrm{M}$ Cantwell, ${ }^{3} \mathrm{~F} \mathrm{~J}$ Bannon, ${ }^{1} \mathrm{~L} \mathrm{~J}$ Murray, ${ }^{2} \mathrm{H} \mathrm{G}$ Coleman. ${ }^{*}$ ${ }^{1}$ Centre for Health Improvement, Queen's University Belfast, Belfast, UK; ${ }^{2}$ Centre for Public Health, Queen's University Belfast, Belfast, UK; ${ }^{3}$ Northern Ireland Cancer Registry, Queen's University Belfast, Belfast, UK

Background Increased incidence of carcinoma in situ (CIS) has generally been attributed to widespread application of mammography screening. However, direct evidence for the impact of screening is rare.

Methods Screen detected and total CIS (ICD-10 D05) incidence data were obtained, between 1990 and 2007 where possible, from regional cancer registries in the UK and the Republic of Ireland (ROI). Age-standardised incidence rates for the UK and ROI, and regional screen detected incidence rates were analysed.

Results The total incidence rate of CIS among women aged 50-64 years increased by 3.1-fold in the UK (1990-2007) and by 2.9-fold in the ROI (1994-2007). An increase of 7.3-fold in the UK and 3.5-fold in the ROI was observed in women aged 65-69 years. Finally, an increase of 8.3-fold in the UK and threefold in the ROI occurred in the age group 70-74 years. Screen detected incidence rates for CIS largely parallelled the total incidence rates for CIS

Conclusion Our results show increased incidence of CIS in all age groups investigated, with the greatest increase observed in women over 65 years. Our regional screen detected incidence rates indicate that increased incidence may partly be attributed to screening. However, other contributing factors must also be considered for the apparent increase in CIS incidence outside the screening age and the consistent difference observed between the screen detected rate and the total incidence rates for CIS.

\section{P1-164 EFFECTIVENESS OF A CLINICAL DECISION SUPPORT SYSTEM FOR BREAST CANCER SCREENING: PRELIMINARY RESULTS IN MAMMOGRAM PERFORMANCE}

doi:10.1136/jech.2011.142976d.57

${ }^{1} \mathrm{~A}$ M G Saldaño, ${ }^{1} \mathrm{D}$ Borbolla, ${ }^{1} \mathrm{C}$ Otero, ${ }^{2} \mathrm{~K}$ Kawamoto, ${ }^{1} \mathrm{~J}$ Vicens, ${ }^{1} \mathrm{~K}$ Kopitowski, ${ }^{2} \mathrm{D}$ Lobach, ${ }^{1} \mathrm{~F}$ G B de Quiros. ${ }^{1}$ Hospital Italiano de Buenos Aires, Ciudad de Buenos Aires, Argentina; ${ }^{2}$ Duke University, Durham, North Carolina, USA

Introduction A decision support system that identifies women lacking results from previous mammograms in their electronic medical record (EMR) and enables immediate corrective intervention was developed. The purpose of this study was to determine if this system increased mammography rates.

Methods In an ongoing cluster randomised pragmatic controlled trial, primary care physicians (PCPs) were allocated to either receive breast cancer screening decision support recommendations in patient's EMR or usual care. PCPs belonging to a Health Maintenance Organization with 150000 members from Buenos Aires and having panels in which at least 100 women were eligible for routine breast cancer screening (no mammogram or a mammogram with BIRADS 0 within the past 2 years). Preliminary results of subjects characteristics and mammograms performed are shown in percentages and compared by $\chi^{2}$ test.

Results A total of 9872 women were eligible, with a mean age of 60.4 (SD 0.57) and were similar in: stroke (1.64\%, p 0.06), 